Témoigner Témoigner. Entre histoire et mémoire

Getuigen Revue pluridisciplinaire de la Fondation Auschwitz

125 | 2017

Histoire et mémoire de la persécution des

homosexuel-le's par les nazis

\title{
À lire/À voir/À suivre
}

\section{(2) OpenEdition}

Journals

Édition électronique

URL : https://journals.openedition.org/temoigner/6745

DOI : $10.4000 /$ temoigner.6745

ISSN : 2506-6390

Éditeur :

Éditions du Centre d'études et de documentation Mémoire d'Auschwitz, Éditions Kimé

Édition imprimée

Date de publication : 1 octobre 2017

Pagination : 165-170

ISBN : 978-2-930953-01-4

ISSN : 2031-4183

Référence électronique

«À lire/À voir/À suivre », Témoigner. Entre histoire et mémoire [En ligne], 125 | 2017, mis en ligne le 24 décembre 2021, consulté le 04 février 2022. URL : http://journals.openedition.org/temoigner/6745 ; DOI : https://doi.org/10.4000/temoigner.6745 


\section{$\checkmark$ À lire/À voir/À suivre}

\section{Les exilés belges en France : histoires oubliées 1914-1918}

Durant la Première Guerre mondiale, une grande partie de la population belge s'est exilée en France. Avec le gouvernement belge installé à Sainte-Adresse, et les 64500 soldats affectés à l'arrière du Front, plus d'un million de réfugiés, dont 10000 enfants installés dans des colonies scolaires, ont traversé l'épreuve de la guerre à l'abri des tirs et du joug ennemi, mais loin de leur terre natale. Ces «Belges du dehors ", accusés d'avoir échappé aux souffrances de l'Occupation, ont vu leur histoire tomber dans l'oubli après la fin du conflit. Certains ont pourtant empêché que des fermes abandonnées tombent en ruine, et permis à la région où ils se sont installés de retrouver une économie dynamique ; beaucoup ont participé à l'approvisionnement du Front en matériel, approvisionnement et équipement

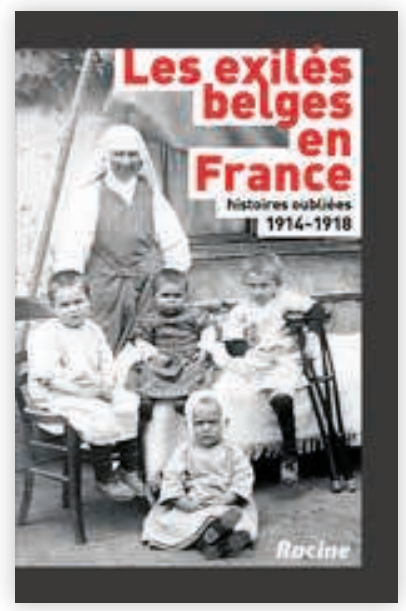

essentiels pour les soldats ; la plupart, enfin, ont fait preuve d'une solidarité exceptionnelle. Àl'heure où la figure du réfugié questionne plus que jamais, ce livre met en lumière l'inventivité et le courage des exilés belges de 14-18.

Les recherches de ces 8 auteurs permettent, ensemble, d'exhumer de l'oubli l'histoire des exilés belges de la Grande Guerre.

\section{$\Leftrightarrow$ Jean-Pierre Popelier, Isabelle Kaanen-Vandenbulcke, Jacques Ganty, Patrick Loodts, Lara Loose, Jean- Philippe Préaux, Mélodie Brassinne, Isabelle Masson-Loodts, Les exilés belges en France : histoires oubliées, Bruxelles, Racine, 2017, 232 p. \\ $\rightarrow$ Recension de cet ouvrage dans notre numéro 126}

\section{Une femme face à l'Histoire}

Pour la dernière fois, image adorée J'ose te caresser en esprit [...]

Reçois, oh lointaine amie,

Les adieux suprêmes de mon cour, Telle une femme au seuil du veuvage

Tels sont les mots que Michel Gorlin adresse à sa femme avant de monter dans le train qui le conduit de Pithiviers à Auschwitz, le 17 juillet 1942. La veille, son épouse Raïssa, née Bloch, a fui la rafle du Vel d'Hiv et mis sa fille, Dora, à l'abri chez des amis. Ces deux jours de juillet 1942 ont scellé son destin. Née en 1898 dans une famille aisée de la bourgeoisie juive de SaintPétersbourg, enthousiasmée par l'aventure bolchevique de 1917, Raïssa fuit à travers l'Europe de l'entre-deux-guerres, de Berlin à

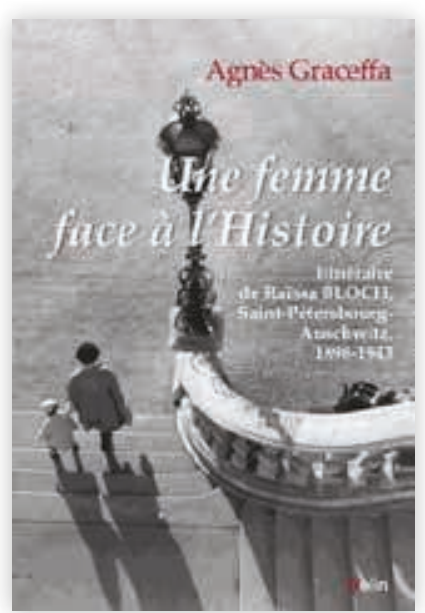

Paris, cherchant avant tout à se consacrer à ses deux passions : l'histoire et la poésie. Son itinéraire est celui d'une exilée qui a rêvé de trouver en France un havre de paix. C'est aussi celui d'une femme qui, par sa seule naissance, voit l'étau de la persécution se refermer sur sa vie. Elle écrit, au seuil du départ de son convoi pour Auschwitz, le 20 novembre 1943 : « Jusqu’à présent, rien ne nous trouble. [...] Au revoir.»

Agnès Graceffa a retracé le parcours bouleversant de cette figure oubliée de l'intelligentsia européenne, historienne du Moyen Âge, poétesse de la lumière, amoureuse passionnée et mère. Ce récit est celui de la lutte d'une femme pour la vie et pour l'art face aux tumultes de l'histoire du XX $\mathrm{X}^{\mathrm{e}}$ siècle.

$\rightarrow$ Agnès Graceffa, Une femme face à I'Histoire, Paris, Belin, novembre 2017, $432 \mathrm{p}$.

Recension de cet ouvrage dans notre numéro 127 
Jorge Semprun. Entre résistance et résilience

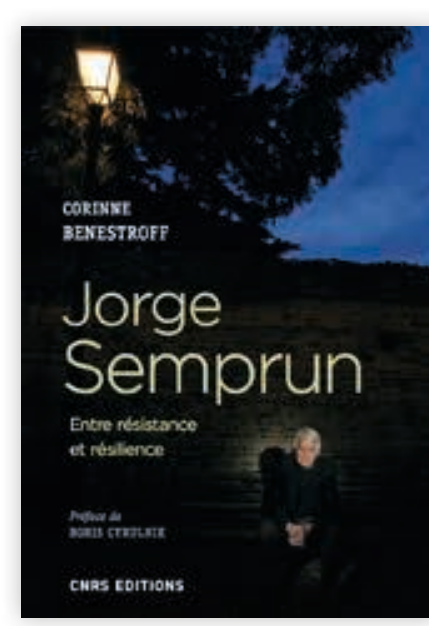

L'œuvre de Jorge Semprun exile, resistant, deporté, écriles territoires de la mort explore es teri XXe siècle et ses désaver ant le XX siècle et ses désastres, arpentant une Europe ravagee, nité, la littérature et la benté monde. Cette ene et la beauté du monde. Cette enquête entrelace littéraire, psychanalyse, critique et paroles vives des archives et paroles vives des témoins. Comnent survit-on à lexpérôle joue ars latirtére ?ue ront cue alors la littérature? Qu qui traversent sones récurrents qui traventson œuvre En royale du témoignage, Semprich royale du témoignage, Semprun transgresse les canons du genre limés de nombreux débats lier d'employé à Buch particucon parcours dogmatique au Part on parours dogmatique au Parti ć́partion espagnol.Chez lui, I séparation fiction/rél est totaleSemprunet sele. On suivra donc de l'enfance au grand âge, Semprun ont l'œuvre inclassable, hommage aux résistants et aux disparus, est leffrution len joulo ments.

$\triangle$ Corinne Benestroff, Jorge Semprun Entre résistance

\section{Algérie. Textes et regards
croisés}

Anne Roche, professeur émérite à l'Université d'Aix-Marseille a l'Université d'Aix-Marseille (France), a publié une vingtaine tha fiction (romans, théatre). et de fiction (romans, théatre). publé publé par une maison dédition et dans le projet de rendre tectors la que les grands a une part de ce que les gráns auteurs d Algér.e $\rightarrow$ Algérie. Textes et regards croisés,

Alger, Casbah, 2017, $390 \mathrm{p}$

$\Leftrightarrow$ Recension de cet ouvrage dans notre numéro 126

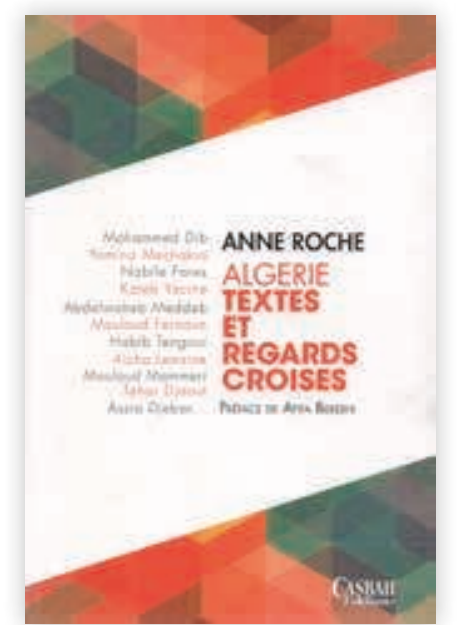

\section{Dossin. Lantichambre}

d'Auschwitz
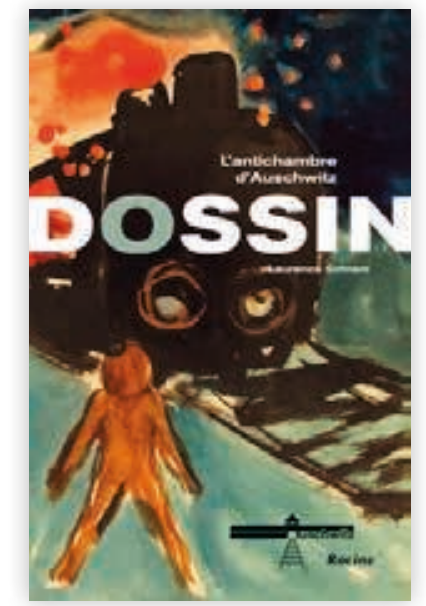

De 1942 à 1944, 25000 Juifs et 350 Tsiganes sont déportés de la Caserne Dossin (Malines) à Auschwitz-Birkenau. Comme Drancy ou Westerbork, la fonction de ce rouage essentiel de la Shoah consiste à rassembler les déporté raciaux en vue de leur déportation génocidaire. Un voyage sans retour ur $95 \%$ d'entre eux.

Pour la première fois, l'histoire de ce camp de rassemblement fai lobjet dune étude scientifique approfondie, menée à la maniere dune enquête policiere. Des des témoignages, des dépositions judiciaires et des illustrations mettent en lumière los parcours detous les acteurs en prés SS, qui rèonenten mâtres a

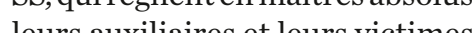
leurs auxiliaires et leurs victine Juives et tsignes.

Laurence Schram analyse tous les aspects de la vie quotidienne dessinjuét dans les trains quiles déportent. Le fonctionnement du camp, la faim, le manque d'hygiène, les mauvais (a) résistance, la situation particulière des Tsiganes, l'organisation des convois, la libération du camp. sont autant de thèmes que le leccont autant de thèmes que le lecouvrage de référence.

Proche collaboratrice de Maxime Steinberg, Laurence Schram a parce Schrama parde la Déportation et de la Résistance (1995), où elle a assuré la tirection directiondre détudedocumentaBelgique, elle est aujourdhi en Belgique, elle est aujourd'hu Senior documentation du ausée Kazerne Dossin. Son doctorat consacré â15) a recu la Prix Na Dossin (2015) a reçu le Pix Natan Ramet J le Prix Fondation Auschwitz

$\rightarrow$ Laurence Schram, Dossin.

$\otimes$ Laurence Schram, Dossin.
L'antichambre d'Auschwitz, Bruxelles,

$\diamond$ Recension de cet ouvrage dans

\section{Lettres à Khayè. Corres-} pondance clandestine d'un amour en temps de guerre

Depuis qu'il a été interné au camp de Pithiviers, en mai 1941, Zysman ecrit presque tous les jours à rédigées en français, mais cen rédigées en français, mais censuen, etpuisles autres, cellesecrites en yiddish, leur langue maternelle, et assées sous le manteau, au nez et à la barbe des autorités concen-

Ces lignes serrées clament son amour absolu pour Khayè,sa «chère anne lumineusen, mais racontent et l'âpre quotidien... Zysman, maintenu dan sert prochain, sions et pressent à de nombreuse reprises toute l'bises toute pleur du projet pleur du proje nazi. Pourtant inlassablement, $i$ ente detransmettreà sonépouse formidable envie de vivre, sa force a détermination.

Débordante de vie et d'amour, cette correspondance inédite dessine le portrait d'un couple malmené pa postore et la folie des hommes pour, finalement, nous donner de courage.

$\triangle$ Zysman Wenig, Lettres à Khayè. Correspondance clandestine d'un amo Lévy, 2017, $296 \mathrm{p}$.

$\diamond$ Recension de cet ouvrage dans

\section{Cahier de mémoire, Kigali,}

\section{4}

Du 7 avril au 3 juillet 1994, le génocide des Tutsi au Rwanda a fait près dur décrites, ana ót été connues, iens : ún cine de visinasto"réns : «génocide de voisinage "gènocide de proximité», «gènoprésent la voix des Mais jusqua présent, la voix des rescapés sest peu fait entendre, à lexception

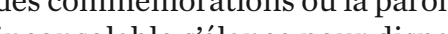
râtre à nouvelu et reur dispasoi, réveillant les traumas.
Les rescapés réunis dansl'atelier de memoire de la Maison de quartie Rwanda avenir à Kigali, ont écrit ou enregistre leurs récits, enplis deur quils considerent comne 'bistoire du sénocide qu'ils ont vécue résister au ńc quils on donner les preuves d'un génocide dont les pŕmices remontent dontes prénices rént années soixante. Acette époque,les feur cahier de mém anteurs leur cahier de 8 ans. Ils découvrent la peur et les persecutions san en connaitre ni

Tout au long des années qui précèdent et notamment en 1973, en Jusqu'en avil 1994, où las, lexil. Jusquen avril1904, où lassassinat de tous les leurs se déroule sous lieu dans la chasse à lhomme moindres recoins des dans les "Où vas-tu? ? leur demandent clles ceserenter refuge. Avant meme d'entendre voisins les renvoient à la mort : Aujourd'hui ce n'est pas comme hier prends ton enfant et pars d'ici." «́́coute Annonciata sœur, tu as été ma voisine nous avons été de grandes amies, mais ici ce n'est pas un refuge pour les 
Tutsi. Même Dieu vous a lâchés.» Cet ouvrage rassemble les récits de quinze rescapés, hommes, femmes, jeunes et personnes âgées. Chaqu serine ens textes auxautresparticipant. Ils se sont accompagnés tout aulong coration Ce sont quinze liviese cest cest un seul livre, celui de celles et Trente-quatre án d'hure Trente-quatre annes dhumilia$\diamond$ Florence Prudhomme (dir.), Cahier de mémoire, Kigali, 2014, Paris,

Carnets de clandestinite de

Carnets de clandestinité de 1942-1943

À 16 ans, Moshé Flinker fuit les Pays-Bas avec ses parents, ses cinq
sœurs et son jeune frère pour tenter d'échapper aux persécutions ter d'èch

nazies. Arrive à Bruxelles, désœuvrè rères, il commence à écrire en hébreu son Journal dans lequel il retranscrit des scènes de la vie quotidienne et suit très attentivement l'évolution de la guerre. analyse l'histoire juive et, anime d'une foi profonde, acquiert la conviction que la création d'un État sur la terre ancestrale est la seule réponse possible à une tenlhistore Ilomprend ausique I'histoire. Il comprend aussi que la connaissance de la langue arabe est te ế funt tence future en Eretz Israel et se (2)

L'un de ses écrits se termine par ces mots : «J'ail'’impression dêtre mort. Me voici.» Arrêté, suite 19 mai 1944 à Auschwitz avec se parents et deux de ses sœurs. En octobre 1944, son père et lui d'Auschwitz vers le camp de Stutthof situé Stutthof situé aux environs de Dantzig, au nord de la Pologne. En ils sont transférés à Echter-

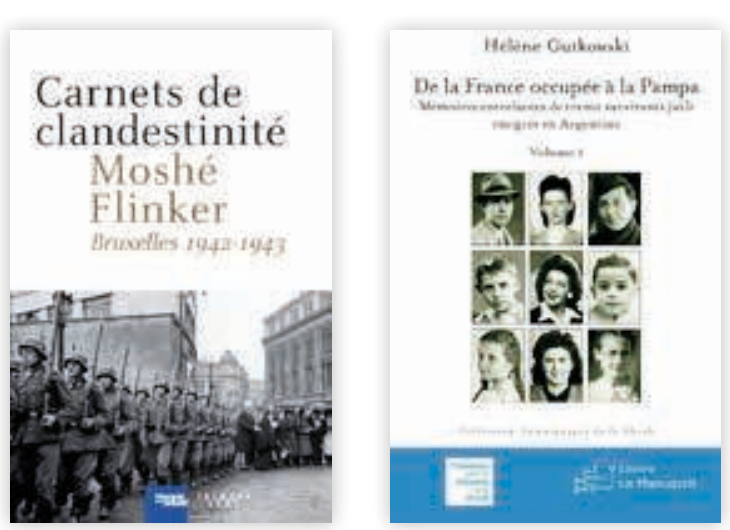

dingen, près de Stuttgart doù ils

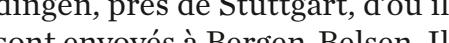
yelsen. I yarnentépuisés, le 22 janvie trouvés à Bruxelles par se sœurs apres la guerre, dans 1 cave de limmeuble où avait vécu force et acuité les angoisses itue et acuité les angoisses spid'une ét dun jeune garçon juif, dene éton iluet politique. Pus encor armes despritsura forcebre. Moshe $\Leftrightarrow$ Moshé Flinker, Carnets de clandestinité : Bruxelles 1942-1943,
Paris, Calmann-Lévy, « Mémorial de la Shoah ", 2017, 176 p.

\section{De la France occupée a la Pampa. Mémoires} lacées de trente survivants juifs émigrés en Argentine

Ce premier volume consacré a ravail collectifde « France... douce France de notre enfance ?», un groupe de parole néà Buenos Aires, rend compte à travers le parcour neuf de ses membres des multiples formes que prit la persécuThes Juifs dans 1 Hexagone internés, sauveurs d'enfants résistants ou déportés, ils avaient alors entre deux et dix-sept ans. Les lettres, documents et photo qu'ils ont précieusement gardés témoignent des séparations, des déportations, mais aussi de la solicos Français quil les sauverent et le Gutk de lis parts. Helen Gutkowski, elle-même enfant d'écout a nis en decoute et de plume pour evoque le chaleureuxeres où les souvenirs fragnentés se confronterent et sunirent pou peindre cette France qui ne fut pas la «douce France» de la chanson. Elle a également pris soin de nou rappoys des juntos du Júron dan le pays des juntes et du péronisme, intelligente introduction qui nous permet dé découvrir cette communaú juive florissante où elle et ses amis-témoins ont pu s'epanouir malgré les incurables meur-

$\diamond$ Hélène Gutkowski, De la France occupée à la Pampa. Mémoires juifs émigrés en Argentine, Paris,
justs Shoah », 2017, 608 p.

$\diamond$ Recension de cet ouvrage dans darite. Is honorent ici la mémoire Le Manuscrit, «Témoignages de
Varsovie Varsovie. Ils vont sauver les archives de l'oubli

Cette fiction prend naissance dans la résistance du ghetto de Varsovie qui aboutiraà la révolte d’avril 1943 Elle s'appuie sur l'histoire d'Emmanuel Ringelblum, militant social et politique, homme de sciences qui entrepris la rédion tive d'un journal, véritable arecde résis journal, veritable arme lait en fer e dans des bidons de lart en fer enforis sous terre. Ces archives, 27000 pages et documents rassen, és en 1669 dosdites retros aujoul'th partie du Patrim aujourd hi partie du Patrinoine mondial de l'UNESCO.Le personnage central, Mhistorien Emmaà

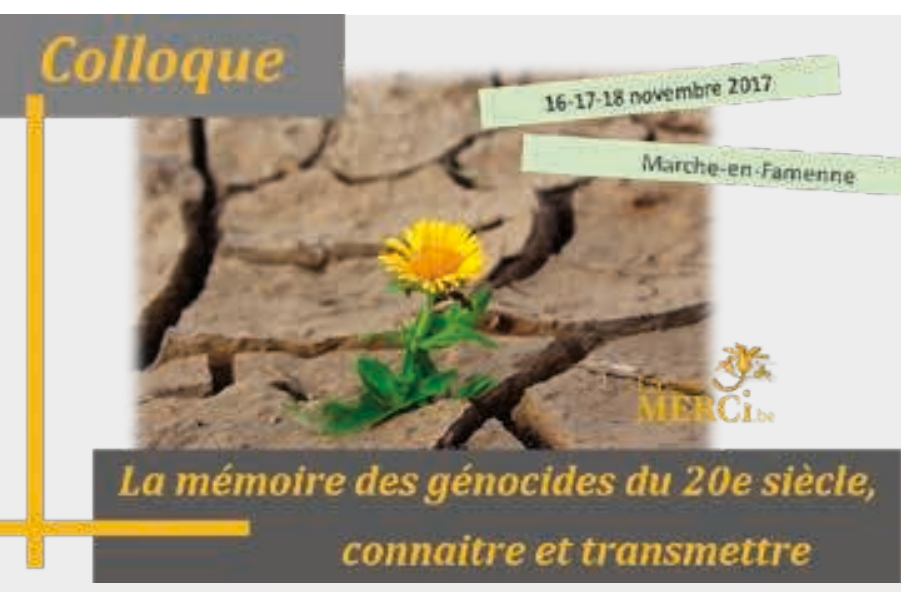

La Fondation $\mathrm{MERCl}$ organise, durant trois journées, un colloque lapproche internationale et interdisciplinaire des génocides du XX siècle. Des orateurs issus de Belgique, du Grand-Duché de Luxembourg et de France aborderont la thématique du point de vue des sciences historiques, juridiques et sociales.

tions et des disponibilités logistiques, des visites de lieux de mémoire et des expositions pourraient être organisées.

PAF : $15 € /$ jour $-35 € / 3$ jours
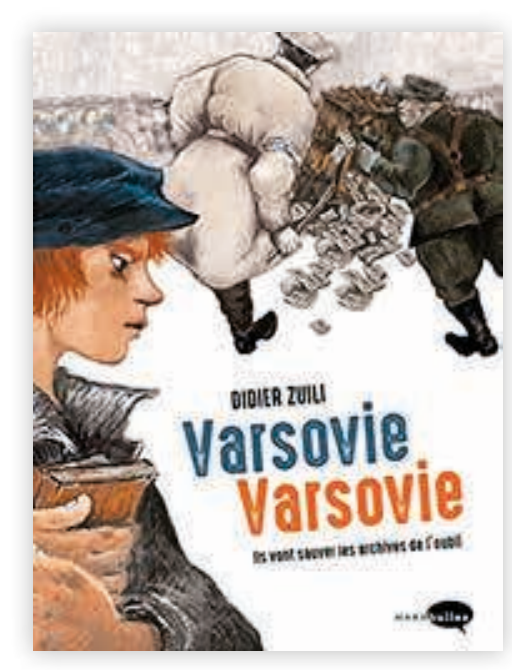

Comment devient-on tortionnaire?

On est d'abord submergé d'épouvante. Duch, chef du camp S-21 au Cambodge à l'époque des Khmer rouges, est responsable delatorture et de la mort dans des conditions Et pourtant, il faut la sidération pour comprendre qui s'est joué entre un individue qui s'est joué entre un individu ne tale et un régime responsable de mortde deuxmillions de person sur une population te personnes C'est le travail auquel s'est livré Françoise Sironi, chargée de l'expertise pioni, chargee de lexpertise psychologique de Duch au Depuis vingt-cinq ans, elle soigne Depuis vingt-cinq ans, elle soigne de massacres, de déportations cées, decime de mortations forcés, de crime de masse. Maisilne suffit pas de prendre en charge les la fabrication des bourreaux, centrer dans leter. "entrer dans leur tête». Comment sont-ils devenus des etres capables du pire? Pour cela, la psy- 
chologie doit se réinventer, se situer à l'intersection de la vie psychique et de la géopolitique. Les Khmers rouges avaient créé l’Angkar, une organisation mystérieuse que chacun devait
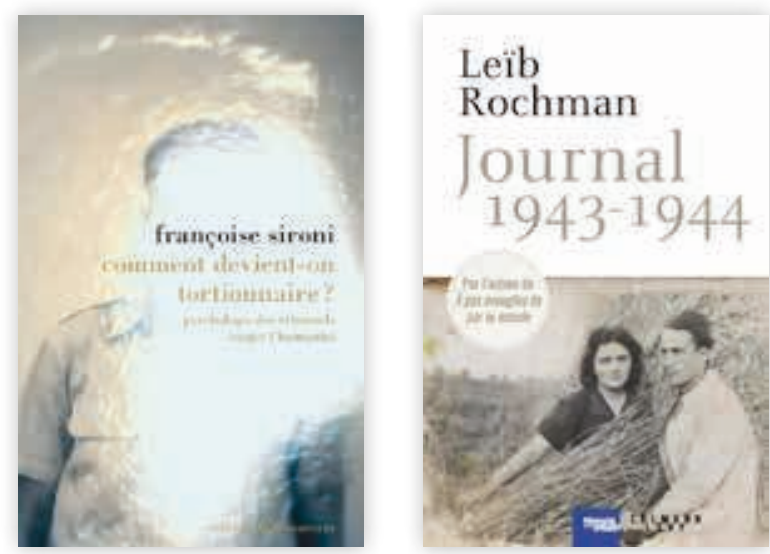

servir et

que l'on nourrissait de sacrifices humains. C'était un « système perpétuel », une théopathie sacrificielle s'épurant en permanence.

Pour déconstruire la mécanique d'un système à la fois psychique, politique et social, Françoise Sironi, grâce aux ressources de la psychologie géopolitique clinique, de l'ethnopsychiatrie et de la schizo-analyse, aux travaux d'Hannah Arendt, de Georges Devereux, Tobie Nathan ou Gilles Deleuze et Félix Guattari, nous donne de nouveaux outils non seulement pour comprendre comment l'impen- sable est arrivé, mais aussi comment déjouer les projets des futurs systèmes criminels susceptibles de nous menacer.

Françoise Sironi, Comment devient-on tortionnaire?, Paris, La Découverte, 31 août 2017, 768 p.

Recension de cet ouvrage dans notre numéro 127

\section{Leïb Rochman, Journal 1943-1944}

Entre 1943 et 1944, Leïb Rochman, auteur du chef d'œuvre À pas aveugles de par le monde, vit caché dans des conditions extrêmement difficiles, quelque part en Pologne. D'abord dans la double cloison d'une cuisine chez une paysanne, avec sa femme, sa belle-sœur et deux amis. Puis dans un grenier et enfin dans une fosse. La voix de Leïb Rochman, incantatoire et douloureuse, décrit la catastrophe qui les oblige à vivre ainsi. Cette souffrance fait écho à la destruction du peuple juif tout entier. En dépit de la peur omniprésente, des maladies, de la faim et du froid, tous continuent d'observer l'essentiel des commandements de la foi juive ; c'est là l'un des aspects les plus poignants de ce témoignage. Rochman dit son aspiration à bâtir une vie nouvelle comme à se reconstruire en tant qu'être humain, dans un lieu où les Juifs seraient enfin les maîtres de leur destin. Un État juif, précise Rochman, en Eretz Israel. Là même où il s'éteindra en 1978.

Leïb Rochman, Journal 1943-1944, Paris, Calmann-Lévy, 2017, 536 p.

$\rightarrow$ Recension de cet ouvrage dans notre numéro 126

\section{SYMPOSIUM : TRADUIRE LE TÉMOIGNAGE - 28 NOVEMBRE 2017}

Les groupes de recherche CLIV (Centrum voor Literatuur in Vertaling - UGent et VUB), CERES (Centre for Reception Studies - KU Leuven) et CMSI (Cultural Memory Studies Initiative - UGent), en collaboration avec la Mémoire d'Auschwitz ASBL, organisent un symposium sur la traduction et le témoignage le 28 novembre 2017. L'événement aura lieu au département de Traduction, d'Interprétation et de Communication de l'Université de Gand.

L'objectif de cette journée consiste à interroger l'importance, l'impact et la difficulté éthique de la traduction de témoignages littéraires et non littéraires aux $X X^{e}$ et $\left.X X\right|^{e}$ siècles. Les communications auront lieu en néerlandais, tandis que le corpus étudié peut être rédigé dans une autre langue. L'accent portera sur des récits de fuite et d'expulsion dans un contexte de guerre ou d'épuration ethnique, des récits d'esclavage ou d'emprisonnement politique sous la dictature, et des récits d'oppression dans un contexte postcolonial. Le volet académique comprendra une série de communications de 20 minutes et une session plénière d'une heure. L'après-midi sera consacré à une table ronde rassemblant des traducteurs de témoignages sur la Shoah vers le néerlandais et le français. 